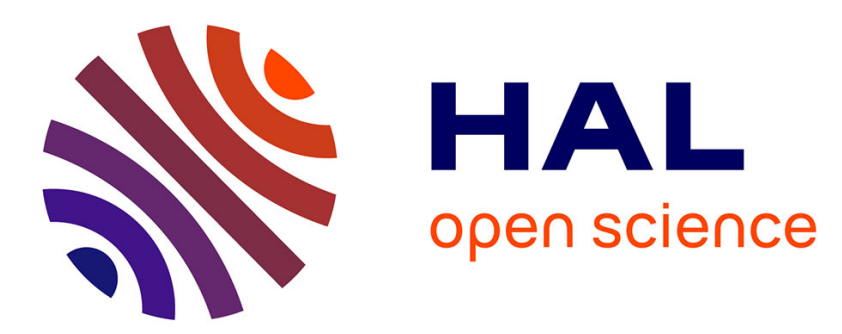

\title{
Direction finding of ELF hiss emissions in a detached plasma region of the magnetosphere
}

M. Hayakawa, N. Ohmi, Michel Parrot, François Lefeuvre

\section{To cite this version:}

M. Hayakawa, N. Ohmi, Michel Parrot, François Lefeuvre. Direction finding of ELF hiss emissions in a detached plasma region of the magnetosphere. Journal of Geophysical Research, 1986, 91 (A1), pp.135. 10.1029/JA091iA01p00135 . insu-03209838

\section{HAL Id: insu-03209838 \\ https://hal-insu.archives-ouvertes.fr/insu-03209838}

Submitted on 27 Apr 2021

HAL is a multi-disciplinary open access archive for the deposit and dissemination of scientific research documents, whether they are published or not. The documents may come from teaching and research institutions in France or abroad, or from public or private research centers.
L'archive ouverte pluridisciplinaire HAL, est destinée au dépôt et à la diffusion de documents scientifiques de niveau recherche, publiés ou non, émanant des établissements d'enseignement et de recherche français ou étrangers, des laboratoires publics ou privés. 


\title{
Direction Finding of ELF Hiss Emissions in a Detached Plasma Region of the Magnetosphere
}

\author{
M. HAYAKAWA ${ }^{1}$ AND N. OHMI \\ Research Institute of Atmospherics, Nagoya University, Toyokawa, Japan \\ M. Parrot and F. Lefeuvre \\ Laboratorie de Physique et Chimie de l'Environnement, Orleans, France
}

\begin{abstract}
Wave normal directions and wave distribution functions of ELF hiss emissions in a detached plasma region of the magnetosphere have been determined, using data from the geostationary satellite GEOS 2 located in the equatorial plane at $L=6.6$. Three different methods of direction finding have been utilized: (1) Means's method based on the hypothesis of a single plane wave, (2) the maximum likelihood method, assuming a few plane waves, and (3) the maximum entropy method of determining the wave distribution function. An intercomparison of the results from those methods has proven that they provide very reliable and definite information on the wave normals. From analyses of two equatorial ELF hiss events it is found that the wave distribution function is composed of a single peak (i.e., the waves have a single propagation direction) and that the wave normals of the ELF hiss originating in the detached plasma region make very small angles with the Earth's magnetic field; also, we were able to make an experimental estimate of the angular width of the unstable cone of the emissions. Both our initial direction finding results in the equatorial plane and the corresponding previous results at higher geomagnetic latitudes are compared with theoretical predictions, assuming that the waves are generated by the electron cyclotron instability due to medium-energy (3-30 keV) electrons. It is concluded that all of the characteristics (morphology and direction-finding results) are consistent with the electron cyclotron instability.
\end{abstract}

\section{INTRODUCTION}

ELF hiss is known to be most commonly observed within the plasmasphere; this is the so-called "plasmaspheric ELF hiss" [Russell et al., 1969; Thorne et al., 1973; Hayakawa and Tanaka, 1977]. However, there is another distinct zone for ELF emissions, namely, the detached plasma regions of the magnetosphere. Detached plasma regions are defined as regions outside the plasmapause where the cold plasma density is considerably enhanced compared with the ambient density [Chappell, 1972]. They are believed to originate in the plasmasphere and to be torn off either by the convection electric field [Chappell, 1974; Barfield et al., 1975] or as a consequence of the interchange instability [Lemaire, 1975]. Several investigators [Chan, 1974; Chan et al., 1974; Chan and Holzer, 1976; Kivelson, 1976; Cornilleau-Wehrlin et al., 1978] have reported that ELF hiss emissions occur in such detached plasma regions, and we refer hereinafter to these emissions as "DP hiss."

Based on OGO 5 measurements, Chan [1974], Chan et al. [1974], and Chan and Holzer [1976] have made detailed investigations of the high correlation between ELF hiss amplitude and plasma density enhancement. They have determined the wave normal directions of many DP hiss events observed at high geomagnetic latitudes $\left(20^{\circ}-50^{\circ}\right)$ and have shown that the wave normals make angles less than $40^{\circ}$ with the earth's magnetic field. Chan [1974] and Kivelson [1976] have tried to test the theoretical model of wave generation of DP hiss by the electron cyclotron instability, using observations such as direction finding results in the off-equatorial region and simultaneous measurements of energetic electron flux.

\footnotetext{
${ }^{1}$ Also at Laboratoire de Physique et Chimie de l'Environnement, Orleans, France.

Copyright 1986 by the American Geophysical Union.

Paper number 5A8742.

$0148-0227 / 86 / 005 A-8742 \$ 05.00$
}

The information concerning the distribution of wave normals in the off-equatorial region as studied by Chan [1974] and Chan and Holzer [1976] is, of course, important. However, they were unable to distinguish between the effects of generation and propagation, since they used only offequatorial results. Hence, information on the wave normals of DP hiss exactly at the magnetic equator is considered to be essential for clarifying the distinction between the generation and propagation effects and also for acquiring evidence on the mechanism of DP hiss because the equatorial plane is thought to be the most likely source region where the wave growth is expected to be largest [Helliwell, 1967; Russell et al., 1969; Tsurutani and Smith, 1977].

The purpose of the present paper is to report the first direction finding studies for a few DP hiss events observed exactly at the magnetic equator, based on data from the geostationary satellite GEOS 2 located at $L=6.6$. Furthermore, three different methods of analysis are applied to the observed events in order to obtain very reliable information on the wave normal directions and wave distribution functions, whereas Chan [1974] and Chan and Holzer [1976] used Means's [1972] method, based on the simple hypothesis of a single plane wave, which was the only possibility at that time. Then the present direction finding results at the equator are compared with those obtained in the off-equatorial region by Chan [1974] and Chan and Holzer [1976], thus allowing us to distinguish between the generation and propagation effects.

The plan of the paper is as follows. In section 2 we give a brief description of the three direction-finding techniques used in the paper, while section 3 is concerned with the characteristics of the DP hiss events studied. The corresponding results on their wave normals are presented in section 4 . Finally, we study the generation and propagation mechanisms of the DP hiss in the light of the present direction-finding results at the equator, together with the corresponding direction-finding data acquired off the equator and in the ionosphere. 


\section{Direction-Finding Techniques}

For the detailed study of electromagnetic waves below the electron gyrofrequency $f_{H}$, several sensor combinations and different modes of on-board data processing have been used on the geostationary satellite GEOS 2 , which is located in the geographic equatorial plane at $L=6.6$ [Jones, 1978; $S$-300 Experimenters, 1979]. Here the data that we are treating were collected in the so-called S-300 "survey mode," and we only use those from three magnetic sensors. The signals are processed by the step frequency analyzer (SFA), which may be connected to any sensor combination, have a bandwidth of $300 \mathrm{~Hz}$, and sweep in frequency over the range $0-77 \mathrm{kHz}$. Before being telemetered to ground, the signals are transposed in frequency, passed through identical low-pass filters cutting off at $450 \mathrm{~Hz}$, and sampled at $1.488 \mathrm{kHz}$ [S-300 Experimenters, 1979].

The wave normal directions are estimated from the SFA data. First, the signals associated with the magnetic components are Fourier-transformed. Then at each frequency that we are concerned with we can estimate a $3 \times 3$ spectral matrix consisting of the mean autopower spectrum of each of the three components taken separately, together with the mean cross-power spectrum of each pair of components [Lefeuvre et $a l ., 1981,1982]$. Three different methods of analysis have been used: (1) Means's [1972] method, (2) the maximum likelihood method of determining the propagation directions of a few plane waves [Buchalet and Lefeuvre, 1981; Hayakawa et al., 1984], and (3) the maximum entropy method of determining the wave distribution function (WDF) [Lefeuvre et al., 1981, 1982]. In the studies by Chan [1974] and Chan and Holzer [1976], they used only Mean's method assuming a single plane wave. The eigenvalues of the observed spectral matrix contain information on the type of wave field or on the propagation model. If there is just a single nonzero eigenvalue, the electromagnetic field is that of a single plane wave. If there are two nonzero eigenvalues, the field consists of two plane waves propagated with two different wave normal vectors. Finally, if the three eigenvalues are of the same order, the field is the sum of three or more plane waves. In the first case, Means's method and the maximum likelihood method assuming one direction of propagation must obviously be chosen. In the third case, one cannot avoid using an approach of the WDF type. In between, i.e., in the second case, one could apply either the maximum likelihood method assuming two directions, or the WDF technique. The simultaneous use of the different methods will yield trustworthy direction-finding results.

In what follows, we adopt a Cartesian coordinate system $O_{x y z}$ where the $z$ axis is parallel to the earth's magnetic field $\tilde{B}_{0}$, the axis $O_{x}$ is in the magnetic meridian plane and is directed away from the earth, while $O_{y}$ completes the orthogonal set and is directed eastward. The wave normal direction $(\tilde{k})$ is characterized by the polar angle $\theta$ between $\tilde{k}$ and $\tilde{B}_{0}$ and by the azimuthal angle $\phi$, the origin of which is $O_{x}$ [Lefeuvre et al., 1982; Hayakawa et al., 1984]. We should also mention that the estimated propagation direction is ambiguous because we use only the magnetic field components for the direction finding.

\section{Characteristics OF THE DP Hiss}

The specific day of December 19, 1979, was chosen by Hayakawa et al. [1984] for the detailed analyses of magnetospheric chorus emissions. The chorus emissions on this day are found to be associated with successive substorms and to take place in the morning and premidnight hours (i.e., LT $=0100-1400$, and 2200-2400). During the LT sector between two intervals, i.e., $L T=1500-2300$ there were no emissions, except at $\mathrm{UT}=1800-1900$ (or LT $=2000-2100$ ) when ELF hiss emissions were detected. The electron density is measured by the mutual impedance experiment [Decreau et al., 1978], and the temporal variation of the measured plasma frequency on that day is presented in Figure 1. As compared with Figure 1, ELF hiss emissions are observed, in association with the enhanced cold plasma density; this has already been discussed in detail by Chan et al. [1974], Kivelson [1976], and CornilleauWehrlin et al. [1978]. The density enhancement at UT $=18$ 19 hours is obviously a detached plasma region because'it appears at $\mathrm{LT} \cong 20$ hours and in the recovery phase of geomagnetic disturbances, which agrees well with the morphology of detached plasma regions established by Chappell [1972, 1974]; furthermore, it is correlated with ELF hiss. The correlation of the fluctuations in the wave field with those in the density as studied by Chan [1974] is not discussed in the present paper.

Figure 2 is a typical spectrogram of ELF emissions observed inside the detached plasma region; it indicates that they are of the hiss type. The SFA data at the times 1800:46.013 UT and 1801:08.030 UT have been selected for the subsequent direction-finding studies, and the power spectra of the DP hiss at those two times are shown in Figures $3 a$ and $4 a$, respectively. The wave intensity in Figure $3 a$ is peaked at $\sim 160 \mathrm{~Hz}$ and thereafter decreases sharply with increasing frequency. The peak power spectral density is $\sim 10^{-5}$ $\gamma^{2} / \mathrm{Hz}\left(\sim 3 \mathrm{~m} \gamma /[\mathrm{Hz}]^{1 / 2}\right)$, and the half-power bandwidth is about $150 \mathrm{~Hz}$. Similar characteristics are also recognizable in Figure $4 a$, except for the smaller peak power spectral density of $\sim 4$ $\times 10^{-6} \gamma^{2} / \mathrm{Hz}$ and the shift of the peak to a lower frequency. These spectral characteristics of the DP hiss in our case seem to be very typical of DP hiss as studied in detail by Chan [1974]. The center frequency of the DP hiss is significantly lower than that of the plasmaspheric ELF hiss discussed by Thorne et al. [1973]

\section{Wave Normal Directions and WaVe Distribution Functions}

In the work of Chan [1974] and of Chan and Holzer [1967] these authors selected the events with sufficient signal-to-noise ratio and sufficiently high coiherence to validate the fundamental supposition of a single plane wave in Means's method. For comparison, the frequency dependence of the coherence is also shown in Figures $3 b$ and $4 b$. For the first event, in Figure $3 b$, the coherence is greater than 0.8 over a rèlatively wide frequency range from $\sim 100$ to $\sim 350 \mathrm{~Hz}$, while the second event (Figure $4 b$ ) we observe coherence larger than 0.8 in a narrow band from $\sim 100$ to $\sim 250 \mathrm{~Hz}$. In the frequency range where coherence exceeds 0.8 the power spectral density of the DP hiss is greater than $5 \times 10^{-7} \gamma^{2} / \mathrm{Hz}$, and we have performed the direction finding at those frequencies so as to have definite answers.

Before carrying out the direction finding based on the maximum likelihood method, we have to determine the propagation model (one- or two-direction model) by means of the eigenvalues $\lambda_{1}, \lambda_{2}$, and $\lambda_{3}$ (ranked in decreasing magnitude) of the spectral matrix observed at each frequency. The criterion for distinguishing between the one- and two-direction models is somewhat arbitrary: We use, on some occasions, a combination of the two ratios, $\alpha=\lambda_{2} / \lambda_{1}$ and $\beta=\lambda_{3} / \lambda_{2}$, the thresholds for which are chosen according to the degree of confidence required [Buchalet and Lefeuvre, 1981; Hayakawa et al., 


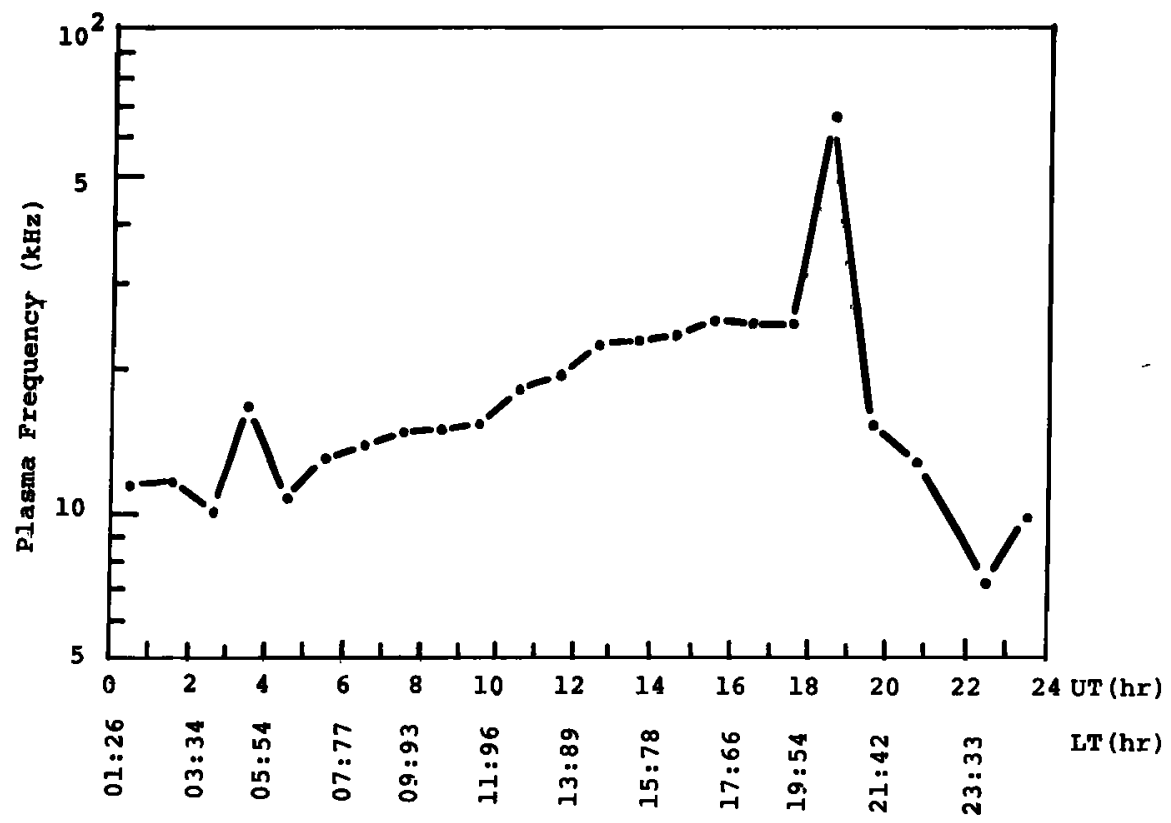

Fig. 1. Temporal variation of the electron plasma frequency $f_{p}$ indicating the equatorial electron density at $L=6.6$, on December 19, 1979. The value of $f_{p}$ at the beginning of each UT hour is plotted. ELF hiss is recorded at UT $=1800-$ 1900 when the satellite is located in a detached plasma region.

1984], while in other cases, the degree of polarization $p=$ $\lambda_{1} /\left(\lambda_{1}+\lambda_{2}+\lambda_{3}\right)$ is used [Lefeuvre et al., 1982; Hayakawa et al., 1985].

First, we will determine the wave normal directions for the first event, illustrated in Figure $3 a$. Analyses of the observed eigenvalues have yielded that the ratio $\alpha=\lambda_{2} / \lambda_{1}$ is smaller than 0.1 at all frequencies in the range where the power spectral density exceeds $5 \times 10^{-7} \gamma^{2} / \mathrm{Hz}$, and hence, following the criterion adopted by Hayakawa et al. [1984], the wave fields at those frequencies are doubtless composed of a single plane wave. Hence Means's [1972] method is applicable, and also the maximum likelihood estimation procedure assuming a one-direction model can be used; the results obtained by both methods are summarized in Table 1. Even though the lowest frequency of $186 \mathrm{~Hz}$ is well above the lower hybrid resonance frequency $(45 \mathrm{~Hz})$, the effects of the protons are included in the kernels (see Storey and Lefeuvre [1980] for detail of the kernels) for the maximum likelihood estimation. It may be seen from the table that the two methods have yielded wave normal directions in good agreement with each other, which are hence considered to be very trustworthy. Moreover, the wave normals make small angles, less than $26^{\circ}$, with the magnetic field over the frequency range up to $\Lambda \sim 0.2(\Lambda$ is the frequency normalized with respect to the electron gy-

\section{GEOS-S300 Survey Mode $19 / \mathrm{Dec} / 1979 \quad 18 \mathrm{~h}$ 0m 7 sec UT}

Position

\begin{tabular}{|c|c|c|}
\hline \multicolumn{2}{|c|}{ Geographic } & Geomagnetic \\
\hline Lat & $-0.1^{\circ}$ & $-0.9^{\circ}$ \\
\hline Long & $24.0^{\circ}$ & $93.7^{\circ}$ \\
\hline DIST & $6.60 \mathrm{R}_{\mathrm{e}}$ & $L=6.60$ \\
\hline
\end{tabular}

$\mathrm{MLT}=19.54 \mathrm{~h}$

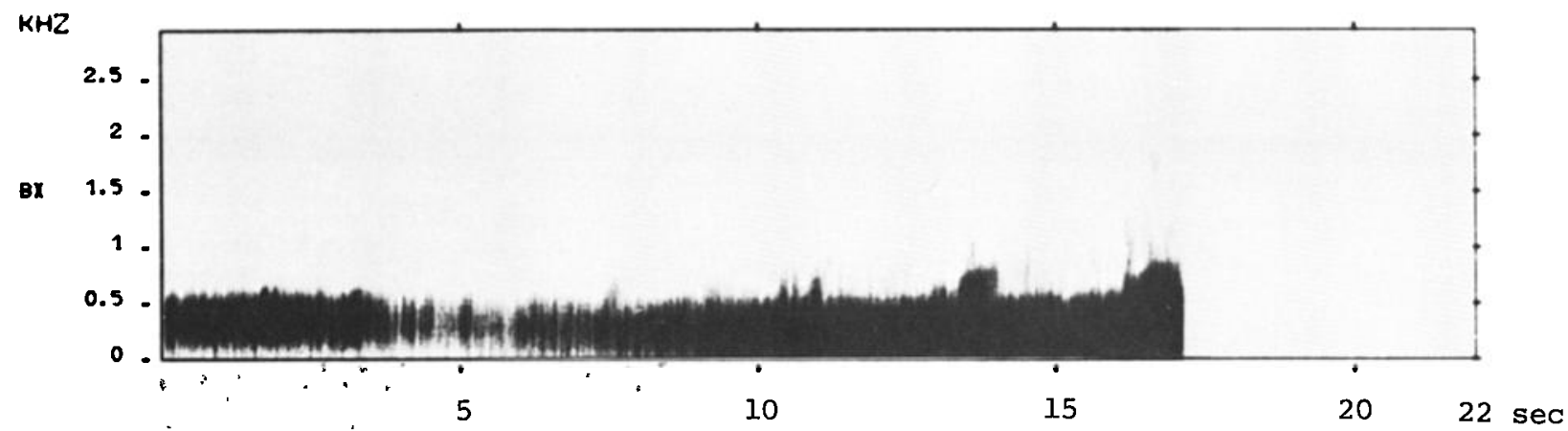

Fig. 2. Frequency spectrum of ELF emissions in the detached plasma region, as measured by a magnetic sensor. 

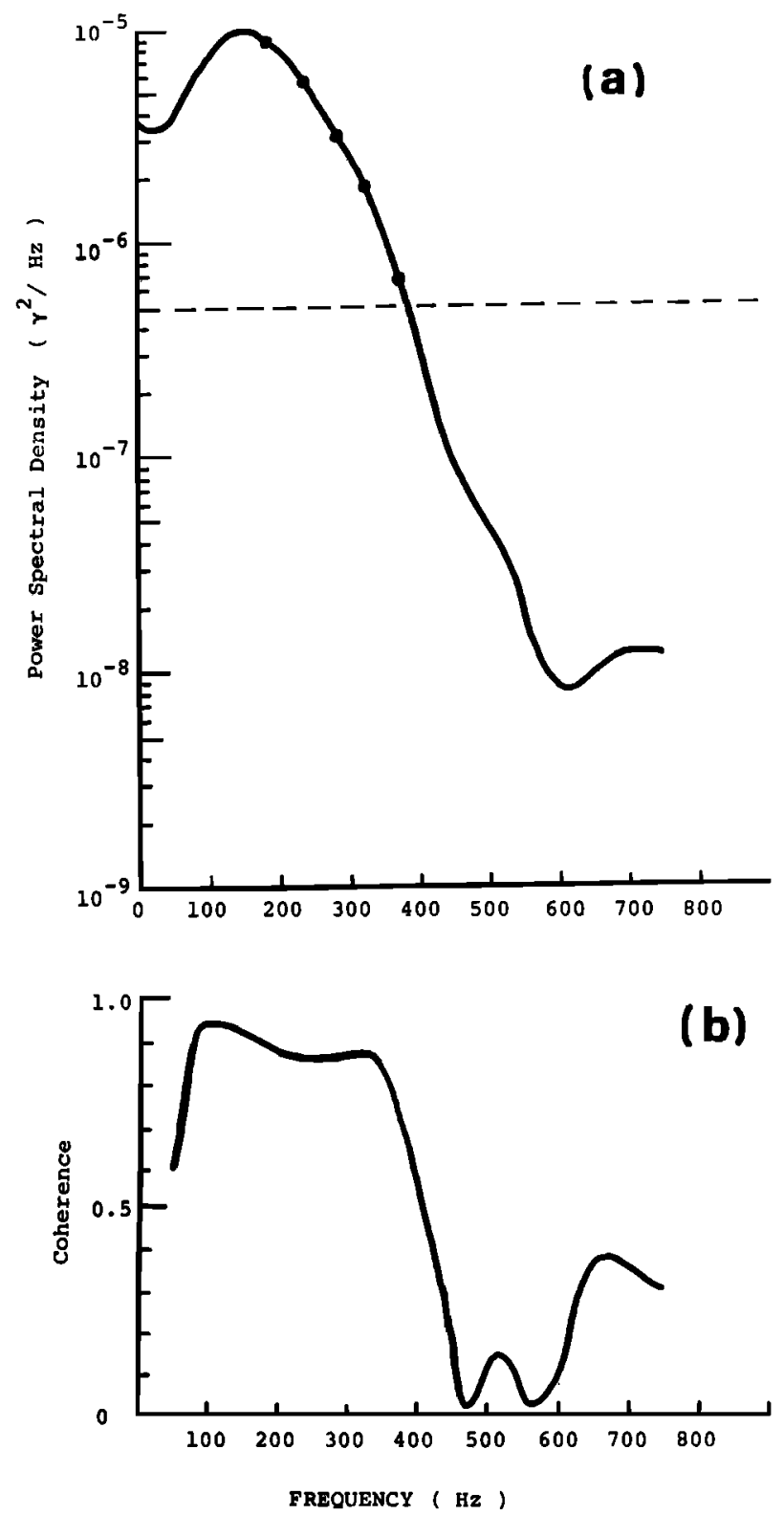

Fig. 3. (a) Frequency dependence of the power spectral density of the DP hiss at 1800:46 UT. At the frequencies indicated by small circles, we have done the direction finding. The power spectral density of $5 \times 10^{-7} y^{2} / \mathrm{Hz}$ is indicated by a horizontal dotted line as a reference level. (b) Corresponding frequency dependence of the coherence.

rofrequency), and there seems to exist an additional tendency for the angle $\theta$ to increase with increasing frequency, from $\sim 8^{\circ}$ at $\Lambda=0.09$ to $\sim 26^{\circ}$ at $\Lambda \sim 0.20$.

Next we discuss the wave normals for the second DP hiss event, illustrated in Figure $4 a$. At the lower frequencies of 186 and $233 \mathrm{~Hz}$, the $\alpha$ value is estimated to be less than 0.1 , so it is certain, according to the criterion of Hayakawa et al. [1984], that the wave fields at those frequencies are composed of a single plane wave. Table 2 summarizes the wave normal directions estimated by the two methods, namely Means's method and the maximum likelihood method assuming a single plane wave, as for the previous event. Again we see that the two wave normal directions at each frequency are in excellent agreement with each other and that the $\theta$ angles have quite small values such as $11^{\circ}-12^{\circ}$. The corresponding wave distribution function at $233 \mathrm{~Hz}$, estimated by the WDF technique
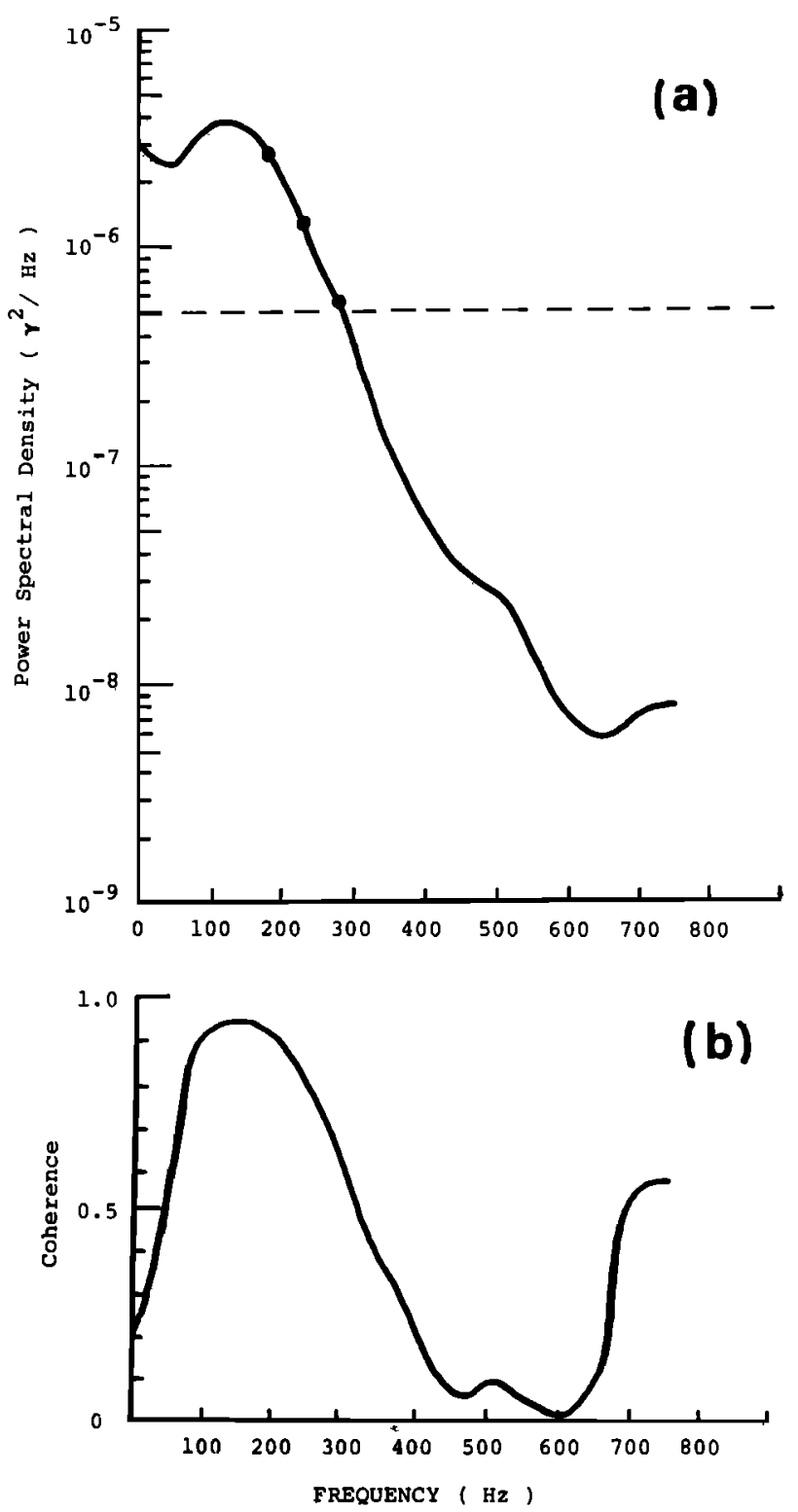

Fig. 4. Same as in Figure 3 but at 1801:08 UT.

[Lefeuvre et al., 1981], is depicted in Figure 5 and is to be compared with the corresponding results at $279 \mathrm{~Hz}$ shown in Figure 6. However, the eigenvalues of the spectral matrix at the higher frequency of $279 \mathrm{~Hz}$ reveal that the $\alpha$ value is 0.131 , not quite satisfying the criterion for a one-direction model formulated by Hayakawa et al. [1984], so we are obliged to rely primarily on the WDF method. The WDF is illustrated in Figure 6, and it does indeed consist of a single narrow peak. Thus, since the resemblance to a single plane wave is con-

TABLE 1. Wave Normal Directions for the DP Hiss in Figure $3 a$

\begin{tabular}{|c|c|c|c|c|c|}
\hline \multirow{2}{*}{$\begin{array}{c}\text { Frequency, } \\
\mathrm{Hz}\end{array}$} & \multirow{2}{*}{$\begin{array}{l}\text { Normalized } \\
\text { Frequency }\end{array}$} & \multicolumn{2}{|c|}{ Means's Method } & \multicolumn{2}{|c|}{$\begin{array}{c}\text { Maximum Likelihood } \\
\text { Method }\end{array}$} \\
\hline & & $\theta$ & $\phi$ & $\theta$ & $\phi$ \\
\hline 186 & 0.096 & $7.7^{\circ}$ & $31.1^{\circ}$ & $9.3^{\circ}$ & $36.0^{\circ}$ \\
\hline 233 & 0.120 & $10.8^{\circ}$ & $17.6^{\circ}$ & $12.5^{\circ}$ & $20.9^{\circ}$ \\
\hline 279 & 0.143 & $17.7^{\circ}$ & $155.6^{\circ}$ & $18.8^{\circ}$ & $161.2^{\circ}$ \\
\hline 326 & 0.168 & $19.5^{\circ}$ & $148.9^{\circ}$ & $20.2^{\circ}$ & $153.2^{\circ}$ \\
\hline 372 & 0.190 & $26.7^{\circ}$ & $143.3^{\circ}$ & $26.3^{\circ}$ & $146.0^{\circ}$ \\
\hline
\end{tabular}


TABLE 2. Wave Normal Directions for the DP Hiss in Figure 4a

\begin{tabular}{|c|c|c|c|c|c|c|c|}
\hline \multirow{2}{*}{$\begin{array}{c}\text { Frequency, } \\
\text { Hz }\end{array}$} & \multirow{2}{*}{$\begin{array}{l}\text { Normalized } \\
\text { Frequency }\end{array}$} & \multicolumn{2}{|c|}{ Means's Method } & \multicolumn{2}{|c|}{$\begin{array}{l}\text { Maximum Likelihood } \\
\text { Method }\end{array}$} & \multicolumn{2}{|c|}{$\begin{array}{l}\text { Maximum Entropy } \\
\text { Method }\end{array}$} \\
\hline & & $\theta$ & $\phi$ & $\theta$ & $\phi$ & $\theta$ & $\phi$ \\
\hline 186 & 0.096 & $10.7^{\circ}$ & $301.9^{\circ}$ & $10.9^{\circ}$ & $292.4^{\circ}$ & $\ldots$ & \\
\hline 233 & 0.120 & $11.8^{\circ}$ & $271.2^{\circ}$ & $11.9^{\circ}$ & $271.7^{\circ}$ & $13.8^{\circ}$ & $261.6^{\circ}$ \\
\hline 279 & 0.143 & $17.1^{\circ}$ & $73.5^{\circ}$ & $14.7^{\circ}$ & $82.6^{\circ}$ & $21.0^{\circ}$ & $83.5^{\circ}$ \\
\hline
\end{tabular}

firmed by the WDF method, it is interesting to compare the WDF result with those obtained by the previous two methods, and Table 2 does this. Means's method and the maximum likelihood method have yielded very similar answers at the two lowest frequencies, but they have yielded wave normals slightly different from each other at $279 \mathrm{~Hz}$. The direction of the peak of the WDF is indicated in the table, and again the results from the WDF method are consistent with those obtained by other methods. The WDF's in Figure 5 and 6 are found to be very circular, but the angular width of the distribution increases with increasing frequency.

\section{Summary OF THE EXPERIMENTAL Results AND Discussion of THE GenERation Mechanism}

Based on the present analyses of the DP hiss events observed at the equator (the geomagnetic latitudes for the two analyzed events are $-0.1^{\circ}$ ) on the GEOS 2 satellite, we can summarize with the following experimental facts for the DP hiss:

1. The detached plasma region detected in the equatorial region at $L=6.6$ by GEOS 2 is found to have occurred during the recovery phase of a substorm and in the LT sector of $\sim 2000$, and it is highly correlated with ELF emissions.

2. The frequency of maximum intensity for the DP hiss ranges between 100 and $200 \mathrm{~Hz}$, which is considerably lower than the corresponding frequency for plasmaspheric ELF hiss.

3. The simultaneous use of three different methods has proved to be a way of obtaining reliable information on the wave normal directions and wave distribution functions.

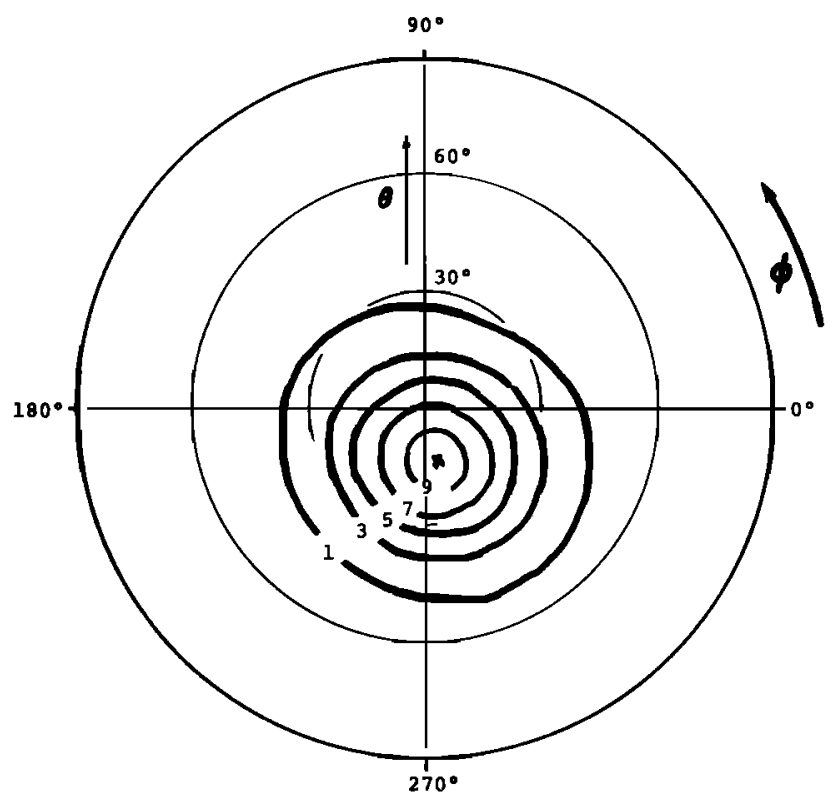

Fig. 5. Wave distribution function as a contour map, for DP hiss at $233 \mathrm{~Hz}$, with its peak indicated by a cross. (The stability parameter $Q$ is 0.54 , and the prediction parameter $P$ is 1.68 ; see Lefeuvre et al. $[1981,1982]$ for the definitions of $P_{r}$ and $Q$.) The half-width of the peak is about $35^{\circ}$.
4. The direction-finding results have shown that the wave distributions consist of a single peak (or that the waves have a single propagation direction) and that the wave normal directions make small angles (less than $25^{\circ}$ ) with the Earth's magnetic field.

5. The wave distribution functions may provide information about the angular width of the unstable cone. The WDF is approximately circular, and the "half-width of the unstable cone" (defined as the range in $\theta$ angle from the peak to the edge labeled 1 in the WDF's) increases with increasing frequency, for instance from $\sim 35^{\circ}$ at $\Lambda=0.12$ to $\sim 50^{\circ}$ at $\Lambda \cong 0.15$.

The morphological properties, items 1 and 2, confirm the results of previous detailed studies by Chan [1974], Chan and Holzer [1976], and Cornilleau-Wehrlin et al. [1978].

The information obtained on the wave normal directions of the DP hiss is of great importance in studying its generation mechanism because the observations were made exactly in the equatorial plane, which is considered to be the most likely source region for the emissions [Helliwell, 1967; Russell et al., 1969; Tsurutani and Smith, 1977]. The hypothesis of local generation at the equator seems to be supported by the experimental fact (item 5) that the WDF's are almost circular, even though the peak is shifted slightly away from the origin. If the relevant waves had propagated over some distance from the source in the nonducted mode, the $\phi$ distribution would be concentrated into the magnetic meridian plane [Thorne, 1969; F. Lefeuvre and L. Cairo, private communication, 1985]. Furthermore, even if we assume ducted propagation from the source and if the detached plasma region were rather elongated in longitude (i.e., if it had a sheetlike structure) [Taylor et

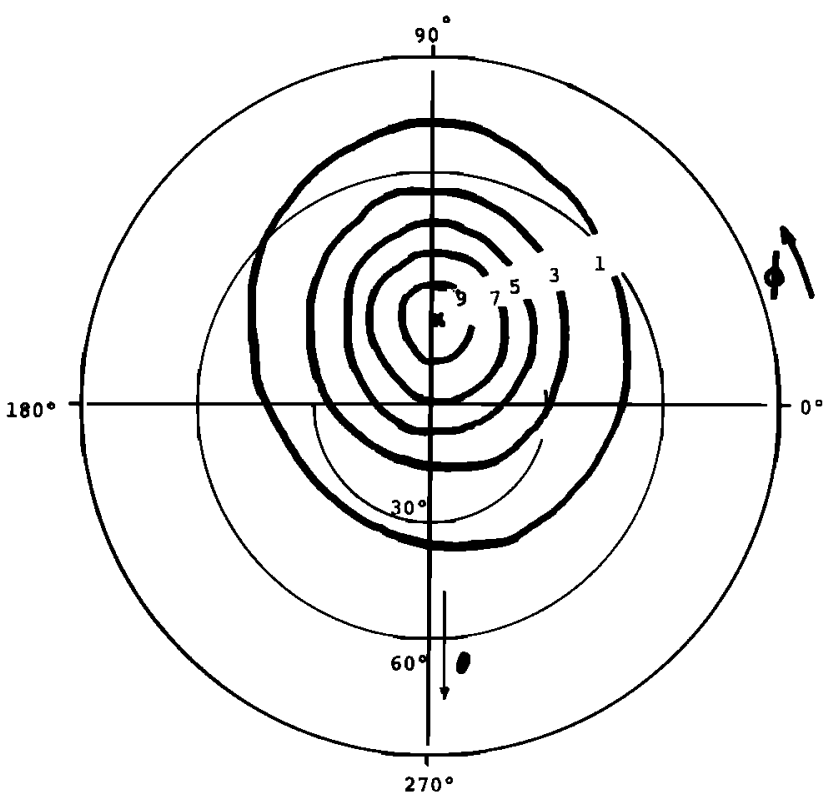

Fig. 6. Wave distribution function at $279 \mathrm{~Hz}\left(Q=0.69\right.$ and $P_{r}=$ 0.93 ); the half-width of the peak is about $50^{\circ}$. 
al., 1970, 1971], we would again expect a tendency for the azimuthal direction to be in the meridian plane. These expectations seem to be in contradiction with the nearly circular shapes of the WDF's. Now, the $\phi$ values of the energy peak at $\phi \sim 90^{\circ}$ (Figure 6) and $\phi \sim 280^{\circ}$ (Figure 5) can be explained by the relative position of the satellite with respect to the source. The small shift in $\theta$ from the origin (14 ${ }^{\circ}$ in Figure 5 and $21^{\circ}$ in Figure 6) might have resulted from the effects of propagation over a short distance, and it is reasonable to consider that circular shapes of the WDF's reflect the properties of the source, that is, they are consistent with isotropic emission generation at the source in the sense that the emission is independent of the azimuthal angle around the magnetic field and depends only on the polar angle.

The direction-finding results given in the present paper (item 4 indicate that wave normals make small angles (less than $25^{\circ}$ ) with the magnetic field. In the study of Chan [1974], if we select the two events observed near the equator $\left(1.4^{\circ}\right.$ and $6.0^{\circ}$ geomagnetic latitude), the wave normals are again found to make small angles (less than $20^{\circ}$ ) which obviously are significantly smaller than those observed at high geomagnetic latitudes. Hence we can conclude that the wave growth at the equator is strongest for the quasi-longitudinal propagation direction $\left(\theta \approx 0^{\circ}\right)$. Burton and Holzer [1974], Hayakawa et al., [1984], and Goldstein and Tsurutani [1984] have reported, similarly, very small $\theta$ (less than $20^{\circ}$ ) for chorus in the vicinity of the equator, in the same range of normalized frequency. The present finding of maximum growth rate for quasilongitudinal propagation is in good agreement with the theoretical prediction by Kennel [1966]. He assumed an $E^{-n}$ energy spectrum for the electrons and found that the wave growth is most rapid at $\theta=0^{\circ}$ at the lowest frequencies $(\Lambda \lesssim$ $0.3)$ and furthermore that a $20^{\circ}\left(50^{\circ}\right)$ half-width for the unstable cone corresponds approximately to an $E^{-3}\left(E^{-2}\right)$ spectrum for the hot electron distribution function, with the additional finding that the unstable cone widens with increasing frequency. In our experimental item 5 , the unstable cone halfwidth estimated from the WDF is $\sim 35^{\circ}$ at $\Lambda=0.12$, increasing with increasing frequency, again in agreement with the theory. The half-width of the experimental unstable cone can be satisfactorily interpreted in terms of the average slope of $E^{-2}$ for the hot electron distribution function. However, in the calculations of Kennel [1966], he did not include the effect of cold electrons. Since the half-width of the unstable cone depends on the relative numbers of cyclotron and Landau resonant electrons, addition of cold electrons to the hot electrons makes the unstable cone narrower, and hence the detailed quantitative comparison between the present experiment and theory is a difficult subject at the moment. We have to comment here that there exists, of course, a lower limit to the particle flux for energetic electron distributions that can be unstable to whistler growth, as studied experimentally by $K i$ velson [1976].

The small wave normal angles at the equator are consistent with both wave generation as mentioned above and ducted propagation down to the ionosphere. Chan [1974] has determined the wave normal directions for DP hiss at high geomagnetic latitudes (mainly $30^{\circ}-50^{\circ}$ ) and has found that the $\theta$ show a considerable scatter over an angular range from $2^{\circ}$ to $40^{\circ}$ and that there is not latitudinal dependence. Hence, it is likely that the wave normals at higher latitudes are larger than those at the equator, which may lead us to consider the possibility of scattering of the wave normals in the course of ducted propagation away from the source region [Helliwell, 1965]. The enhancement factor of the detached plasma region in the present paper is extremely high (more than 10 times higher than the ambient density) compared with the values for normal whistler ducts as given by Angerami [1970], so we can expect wave trapping at large $\theta$ angles, which agrees with Chan's observations.

These DP hiss emissions may reasonably be thought to remain trapped by the density enhancement of the detached plasma region right down to the ionosphere. Data from ionospheric satellites enabled Tulunay and Hughes [1973], Taylor et al. [1970, 1971], and Maynard and Chen [1975] to identify the detached plasma density enhancement in the ionospheric trough region. Probably in association with these detached plasma regions, Chan [1974] detected DP hiss on OGO 5, but simultaneous ion density data were not available to him. Based on the simultaneous high-resolution density measurement and the direction finding for ELF hiss made on the AUREOL 3 satellite, Beghin et al. [1985] have recently found a close association between enhancements in local electron density and the appearance of impulsive ELF emissions, exactly as was found on OGO 5 by Chan [1974]; furthermore, those ELF waves are shown to be trapped within the fieldaligned plasma density enhancements, by means of the direction finding results.

For more quantitative studies, the quasi-linear theory has been proposed, extending the theory of the linear electron cyclotron instability. Following Kennel [1966], papers by Roux and Solomon [1971], by Etcheto et al. [1973] and by Sazhin [1984] presented self-consistent calculations, leading to estimates of the frequency and power spectral density at the peak in the whistler spectrum. A dynamic equilibrium is established in which the waves are being continuously generated and particles are continuously injected and are lost by pitchangle diffusion into the loss cone. The stationarity of the process of wave generation is achieved by the balance of total wave amplification along the field line and wave loss accompanying the partial reflection of the waves from the ionosphere. In these theories, quasi-longitudinal $\left(\theta \approx 0^{\circ}\right)$ or ducted propagation is assumed, which is experimentally validated by our direction-finding results at the equator and also by the observations of Beghin et al. [1985] at ionospheric heights. The frequency $f_{\max }$ at which the wave energy is maximal is given by [Sazhin, 1984].

$$
f_{\max }=\frac{c^{2} f_{H}^{3}}{w^{2} 1.2 f_{p}^{2}}
$$

where $c$ is the velocity of light and $w$ is the characteristic velocity of the incoming electrons at the $L$ shell of the wave generation. The corresponding maximum power spectral density $\left\langle B_{f}{ }^{2}\right\rangle_{\max }$ at $f_{\max }$ can be approximated by [Sazhin, 1984]

$$
\left\langle B_{f}^{2}\right\rangle_{\max } \propto n_{e q} L^{5.5} W_{0}^{1.5} L_{0}^{4.5} \frac{d n_{i}}{d t}
$$

where $n_{e q}$ is the equatorial electron density on the $\mathrm{L}$ shell where the waves are generated, $W_{0}$ is the electron energy at $L=L_{0}, L_{0}$ can be chosen arbitrarily, and $d n_{i} / d t$ is the rate of influx of the electrons. The observed peak frequency is used in (1) to estimate the value of the characteristic energy of the incoming electrons, corresponding to $w$, as $3-20 \mathrm{keV}$, taking into account the measured $f_{\mathrm{H}}$ and the measured range of $f_{p}$ of the detached plasma region as seen from Figure 1. Equation (2) implies that the peak power $\left\langle B_{f}{ }^{2}\right\rangle_{\max }$ is directly proportional to $n_{e q} L^{5.5}$ for a given incoming particle distribution and influx rate $d n_{i} / d t$. In our case, $L$ is fixed at 6.6 , so $\left\langle B_{f}^{2}\right\rangle_{\max }$ is proportional to $n_{e q}$. As may be seen from Figure 1, 
$n_{\text {eq }}$ within the detached plasma region is about 10 times larger than that in the ambient plasma, resulting in the observed enhancement of DP hiss.

Finally, we can conclude that all of the observed characteristics and the direction-finding results can be interpreted satisfactorily in terms of the electron cyclotron instability.

Acknowledgments. The authors would like to express their sincere thanks to P. Robert of CRPE (Issy-les-Moulineaux) for supplying the Earth's magnetic field data and to J. Etcheto and B. Higel of CRPE (Issy-les-Moulineaux) and P. Decreau of LPCE (Orleans) for supplying the data-of-plasma frequency measurements from the S-301 and S-304 experiments, respectively. Thanks are also due to CNES (Toulouse) for making the survey mode microfiches, some of which have been reproduced in the present paper. Useful discussion with $\mathbf{J}$. Ohtsu and Y. Tanaka of Nagoya University and C. Beghin of LPCE (Orleans) are appreciated. The present work is partly supported by the International Joint Project of Japan Society of Promotion of Science and also partly by Takeda Science Foundation, to which we are grateful. Finally, the authors are indebted to the referee for his careful reading of the paper and useful comments.

The Editor thanks L. R. O. Storey for his assistance in evaluating this paper.

\section{REFERENCES}

Angerami, J. J., Whistler duct properties deduced from VLF observations made with the OGO 3 satellite near the magnetic equator, $J$. Geophys. Res., 75, 6115, 1970.

Barfield, J. N., J. L. Burch, and D. J. Williams, Substorm-associated reconfigurations of the duskside equatorial magnetosphere, A source mechanism for detached plasma regions, J. Geophys. Res., $80,47,1975$.

Beghin, C., J. L. Rauch, F. Lefeuvre, R. Debrie, J. C. Cerisier, J. J. Berthelier, O. A. Maltseva, and N. I. Massevitch, Experimental evidence of field-aligned ELF plasma ducts in ionospheric trough and in the auroral zone, in Résultats du PROJET ARCAD 3 et des Programmes Récents en Physique de la Magnétosphère et de TIonosphère, p. 517, Cepadues Editions, Toulouse, France, 1985.

Buchalet, L. J., and F. Lefeuvre, One and two direction models for VLF electromagnetic waves observed on board GEOS 1, J. Geophys. Res., 86, 2377, 1981.

Burton, R. K., and R. E. Holzer, The origin and propagation of chorus in the outer magnetosphere, J. Geophys. Res., 79, 1014, 1974.

Chan, K. W., Extremely low frequency hiss emissions in the magnetosphere, Ph.D. thesis, Univ. of Calif., Los Angeles, 1974.

Chan, K. W., and R. E. Holzer, ELF hiss associated with plasma density enhancements in outer magnetosphere, J. Geophys. Res., 81, 2267, 1976.

Chan, K. W., R. E. Holzer, and E. J. Smith, A relation between ELF hiss amplitude and plasma density in the outer plasmasphere, $J$. Geophys. Res., 79, 1989, 1974.

Chappell, C. R., Recent satellite measurements of the morphology and dynamics of the plasmasphere, Rev. Geophys., 10, 951, 1972.

Chappell, C. R., The convergence of fact and theory on magnetospheric convection, in Correlated Interplanetary and Magnetospheric Observations, p. 277, D. Reidel, Hingham, 1974.

Cornilleau-Wehrlin, N., R. Gendrin, F. Lefeuvre, M. Parrot, R. Grard, D. Jones, A. Bahnsen, E. Ungstrup, and W. Gibbons, VLF electromagnetic waves observed onboard GEOS-1, Space Sci. Rev., $22,371,1978$.

Decreau, P. M. E., C. Beghin, and M. Parrot, Electron density and temperature, as measured by the mutual impedance experiment on board GEOS-1, Space Sci. Rev., 22, 581, 1978.

Etcheto, J., R. Gendrin, J. Solomon, and A. Roux, A self-consistent theory of magnetospheric ELF hiss, J. Geophys. Res., 78, 8150, 1973 .

Goldstein, B. E., and B. T. Tsurutani, Wave normal directions of chorus near the equatorial source region, J. Geophys. Res., 89, 2789, 1984.

Hayakawa, M., and Y. Tanaka, ELF emissions observed at Moshiri (L 1.6), Nature Phys. Sci., 270, 703, 1977.

Hayakawa, M., Y. Yamanaka, M. Parrot, and F. Lefeuvre, The wave normals of magnetospheric chorus emissions observed on board GEOS 2, J. Geophys. Res., 89, 2811, 1984.

Hayakawa, M., F. Lefeuvre, and J. L. Rauch, The direction finding aboard Aureol-3 of elf waves at frequency above and below the proton gyrofrequency, in Résultats du PROJET ARCAD 3 et des Programmes Récents en Physique de la Magnétosphère et de I'Ionosphére, p. 499, Cepadues Editions, Toulouse, France, 1985.

Helliwell, R. A., Whistlers and Related Ionospheric Phenomena, Stanford Univ. Press, Stanford, Calif., 1965.

Helliwell, R. A., A theory of discrete VLF emissions from the magnetosphere, J. Geophys. Res., 72, 4773, 1967.

Jones, D., Introduction to the S-300 wave experiments aboard GEOS, Space Sci. Rev., 22, 327, 1978.

Kennel, C. F., Low frequency whistler mode, Phys. Fluids, 9, 2190, 1966.

Kivelson, M. G., Instability phenomena in detached plasma regions, J. Atmos. Terr. Phys., 38, 1115, 1976.

Lefeuvre, F., M. Parrot, and C. Delannoy, Wave distributions estimation of VLF electromagnetic waves observed on board GEOS 1 , J. Geophys. Res., 86, 2359, 1981.

Lefeuvre, F., T. Neubert, and M. Parrot, Wave normal directions and wave distribution functions for ground-based transmitter signals observed on GEOS 1, J. Geophys. Res., 87, 6203, 1982.

Lemaire, J., The mechanisms of formation of the plasmapause, Ann. Geophys., 31, 175, 1975.

Maynard, N. C., and A. J. Chen, Isolated cold plasma regions: Observations and their relation to possible production mechanisms, $J$. Geophys. Res., 80, 1009, 1975.

Means, J. D., Use of the three-dimensional covariance matrix in analyzing the properties of plane waves, J. Geophys. Res., 77, 5551, 1972.

Roux, A., and J. Solomon, Self-consistent solution of the quasi-linear theory: Application to the spectral shape and intensity of VLF waves in the magnetosphere, J. Atmos. Terr. Phys., 33, 1457, 1971.

Russell, C. T., R. E. Holzer, and E. J. Smith, OGO 3 observations of ELF noise in the magnetosphere, 1, Spatial extent and frequency of occurrence, J. Geophys. Res., 74, 755, 1969.

S-300 Experimenters, Measurements of electric and magnetic wave fields and of cold plasma parameters aboard GEOS-1, Preliminary results, Planet. Space Sci., 27, 317, 1979.

Sazhin, S. S., A model for hiss-type mid-latitude VLF emissions, Planet. Space Sci., 32, 1263, 1984.

Storey, L. R. O. and F. Lefeuvre, The analysis of 6-component measurements of a random electromagnetic wave field in a magnetoplasma -II. The integration kernels, Geophys. J. R. Astron. Soc., $62,173,1980$.

Taylor, H. A., Jr., H. C. Brinton, and A. R. Deshmukh, Observations of irregular structure in thermal ion distribution in the duskside magnetosphere, J. Geophys. Res., 75, 2481, 1970.

Taylor, H. A., Jr., J. M. Grebowsky, and W. J. Walsh, Structured variations of the plasmasphere: Evidence of a corotating plasma tail, J. Geophys. Res., 76, 6806, 1971.

Thorne, R. M., Whistler mode propagation in the off-meridian plane, Internal Report, Dep. of Meteorology, Univ. of Calif., Los Angeles, 1969.

Thorne, R. M., E. J. Smith, R. K. Burton, and R. E. Holzer, Plasmaspheric hiss, J. Geophys. Res., 78, 1581, 1973.

Tsurutani, B. T., and E. J. Smith, Two types of magnetospheric ELF chorus and their substorm dependences, J. Geophys. Res., 85, 5112, 1977.

Tulunay, Y., and A. R. W. Hughes, A satellite study of the midlatitude trough in electron density and VLF radio emissions during the magnetic storm of 25-27 May, 1967, J. Atmos. Terr. Phys., 35, 153, 1973.

M. Hayakawa and N. Ohmi, Research Institute of Atmospherics, Nagoya University, Toyokawa, Aichi, 442, Japan.

F. Lefeuvre and M. Parrot, Laboratoire de Physique et Chimie de l'Environnement, 45045 Orleans Cedex, France.

(Received June 25, 1985; revised September 6, 1985; accepted September 6, 1985.) 